\title{
Diagnostic performance of exercise bicycle testing and single-photon emission computed tomography: comparison with 64-slice computed tomography coronary angiography
}

\author{
A. C. Weustink $\cdot$ L. A. Neefjes $\cdot$ A. Rossi $\cdot$ W. B. Meijboom $\cdot$ \\ K. Nieman • E. Capuano $\cdot$ E. Boersma $\cdot$ N. R. Mollet $\cdot$ \\ G. P. Krestin $\cdot$ P. J. de Feyter
}

Received: 15 September 2010/ Accepted: 23 December 2010/Published online: 8 January 2011

(C) The Author(s) 2011. This article is published with open access at Springerlink.com

\begin{abstract}
To conduct a comparison of the diagnostic performance of exercise bicycle testing and singlephoton emission computed tomography (SPECT) with computed tomography coronary angiography (CTCA) for the detection of obstructive coronary artery disease (CAD) in patients with stable angina. 376 symptomatic patients (254 men, 122 women, mean age $60.4 \pm 10.0$ years) referred for noninvasive stress testing (exercise bicycle test and/or SPECT) and invasive coronary angiography were included. All patients underwent additional 64-slice CTCA. The diagnostic performance of exercise bicycle testing (ST segment depression), SPECT (reversible perfusion defect) and CTCA ( $\geq 50 \%$ lumen diameter reduction) was presented as sensitivity, specificity, positive and negative predictive value (PPV and NPV) to detect or rule out obstructive $\mathrm{CAD}$ with quantitative coronary angiography as reference standard. Comparisons of exercise bicycle testing versus CTCA $(n=334)$, and SPECT versus CTCA $(n=61)$ were performed. The
\end{abstract}

A. C. Weustink $(\bowtie) \cdot$ L. A. Neefjes · A. Rossi ·

K. Nieman · E. Capuano · N. R. Mollet ·

G. P. Krestin - P. J. de Feyter

Erasmus MC, Department of Radiology, P.O. Box 2040,

3000 CA Rotterdam, The Netherlands

e-mail: a.weustink@erasmusmc.nl

A. C. Weustink - L. A. Neefjes - A. Rossi -

W. B. Meijboom · K. Nieman · E. Boersma ·

N. R. Mollet · P. J. de Feyter

Erasmus MC, Department of Cardiology, P.O. Box 2040,

3000 CA Rotterdam, The Netherlands diagnostic performance of exercise bicycle testing was significantly $(P$ value $<0.001)$ lower compared to CTCA: sensitivity of $76 \%$ (95\% CI, 71-82) vs. $100 \%$ (95\% CI, 97-100); specificity of 47\% (95\% CI, 36-58) vs. 74\% (95\% CI, 63-82). We observed a PPV of 70\% (95\% CI, 65-75) vs. 91\% (95\% CI, 87-94); and NPV of $30 \%(95 \%, 25-35)$ vs. $99 \%(95 \%, 90-100)$. There was a statistically significant difference in sensitivity $(P$ value $<0.05)$ between SPECT and CTCA: $89 \%$ (95\% CI, 75-96) vs. 98\% (95\% CI, 87-100); but not in specificity ( $P$ value $>0.05): 77 \%$ (95\% CI, 50-92) vs. $82 \%$ (95\% CI, 56-95). We observed a PPV of $91 \%$ (95\% CI, 77-97) vs. 93\% (95\% CI, 81-98); and NPV of $72 \%(95 \%, 46-89)$ vs. $93 \%(95 \%, 66-100)$. SPECT and CTCA yielded higher diagnostic performance compared to traditional exercise bicycle testing for the detection and rule out of obstructive CAD in patients with stable angina.

Keywords Exercise bicycle testing - Single-photon emission computed tomography $\cdot$ SPECT $\cdot$ Computed tomography coronary angiography $\cdot \mathrm{CT} \cdot$ Diagnostic accuracy

\section{Introduction}

Exercise bicycle testing represents a widely available and inexpensive diagnostic modality and was part of the initial assessment in $76 \%$ of patients with 
suspected angina in the Euro Heart Survey [1]. However, exercise bicycle testing is limited in the prediction of adverse events with a reported $47 \%$ of events occurring during follow-up in patients with a negative exercise bicycle test result [2]. Nuclear myocardial perfusion imaging (MPI) using singlephoton emission computed tomography (SPECT) allows the noninvasive assessment of the hemodynamic significance of coronary artery stenoses by the detection of myocardial ischemia and provides complementary information for risk stratification [3].

Computed tomography coronary angiography (CTCA) has rapidly emerged as an alternative noninvasive modality for the diagnosis of CAD. The diagnostic performance of CTCA on a per patient level is high with sensitivities ranging from 93 to $100 \%$ and specificity ranging from 82 to $96 \%$. In particular, CTCA yields a high negative predictive value to reliable rule out of the presence of significant coronary stenosis [4]. Similar to nuclear MPI, recent studies reported the incremental value of CTCA in predicting all-cause mortality in symptomatic patients $[5,6]$.

In this study, we performed a comparison of the diagnostic performance of exercise bicycle testing with CTCA, and SPECT with CTCA, respectively, to detect obstructive coronary artery disease using invasive coronary angiography (ICA) as the standard of reference.

\section{Methods}

Study design

We evaluated 376 symptomatic patients ( 254 men; 122 women, mean age $60.4 \pm 10.0$ ) who were referred for stress testing (exercise bicycle test and/or SPECT) and ICA based on gender, age, type (typical, atypical or nonanginal) and severity of chest pain. Typical angina was defined when the following three characteristics were present: (1) sub-sternal discomfort (2) precipitated by physical exertion or emotion and (3) relieved with rest or nitroglycerine within $10 \mathrm{~min}$. Atypical angina pectoris was defined when only two out of these three symptom characteristics were met. Nonanginal chest pain was defined when only one was met or absence of the described symptoms.

All patients underwent additional CTCA irrespective of the clinical judgment and stress test outcome as part of a running research protocol. The study protocol was approved by the institutional review board of the Erasmus University Medical Center and informed consent was obtained in all patients.

\section{Patient population}

The study population was obtained from July 2004 until September 2008. Patients with acute coronary syndromes, previous history of percutanous coronary stent placement, coronary artery bypass surgery and prior myocardial infarction were excluded from the study.

Specific CT related exclusion criteria were impaired renal function (serum creatinine $>120 \mu \mathrm{mol} / \mathrm{L}$ ), persistent arrhythmias, inability to perform a breath hold of $15 \mathrm{~s}$, or known allergy to Iodinated contrast material.

\section{Exercise bicycle test}

Patients underwent exercise bicycle testing in the absence of contraindications (left bundle branch block, paced rhythm, the Wolff-Parkinson-White syndrome, left ventricle hypertrophy, electrolyte imbalance, intraventricular conduction abnormalities, use of digitalis, or severe aortic stenoses [7]. The exercise bicycle test was interpreted blinded to the CTCA and ICA findings. The exercise bicycle test result was considered positive if the electrocardiogram showed horizontal or down-sloping ST-segment depression or elevation $[\geq 1 \mathrm{~mm}(0.1-\mathrm{mV})$ for $\geq 60-80 \mathrm{~ms}$ after the end of the QRS complex] [7]. The exercise bicycle test result was considered equivocal if ischemic ST depression was absent but heart rate did not reach $85 \%$ of the maximum predicted for age and gender, if nondiagnostic ST-segments were present during exercise (0.5- to 0.9-mm horizontal ST-segment depression, ST-segment depression with slight upslope, baseline ST-segment and T-wave abnormalities with nondiagnostic changes on stress) or exercise capacity was limited [7, 8].

Single-photon emission computed tomography (SPECT)

SPECT image acquisition and reconstruction was performed as described previously [9]. A dose of $370 \mathrm{MBq}$ of 99mTc-sestamibi (Cardiolite; Bristol- 
Myers Squibb Pharma Belgium, Brussels, Belgium) was administered intravenously approximately $1 \mathrm{~min}$ prior to termination of the stress test. The performance of SPECT was evaluated based on achievement of target heart rate $(85 \%$ of the maximum predicted for age and gender); horizontal or downsloping ST-segment depression or elevation $[\geq 1 \mathrm{~mm}$ $(0.1-\mathrm{mV})$ for $\geq 60-80 \mathrm{~ms}$ after the end of the QRS complex]; severe angina or clinically important changes in blood pressure or heart rhythm.

For studies performed with the patient at rest, $370 \mathrm{MBq}$ of sestamibi was injected at least $24 \mathrm{~h}$ after the stress test. Single-photon emission computed tomography (SPECT) images were acquired with a Gammasonics single-head Rota camera (Orbiter; Siemens, Iselin, NJ) without attenuation or scatter correction, by using a low-energy all-purpose collimator. Image acquisition and reconstruction were performed as earlier described [9]. The SPECT images were interpreted blinded to the CTCA and ICA findings. A reversible perfusion defect was defined as a perfusion defect on stress images that partially or completely resolved at rest. A fixed perfusion defect was defined as a perfusion defect on stress images that persisted on rest images. Findings were considered as abnormal in the presence of a fixed and/or reversible perfusion defect.

Computed tomography coronary angiography (CTCA)

The first enrolled patients $(n=119)$ underwent 64-slice CTCA (Sensation 64, Siemens Healthcare, Forchheim, Germany) from October 2004 to March 2006. Patients with a heart rate exceeding $65 \mathrm{bpm}$ received either additional oral or intravenous betablockers. The subsequent 257 patients underwent dual-source CTCA (Definition, Siemens Healthcare, and Forchheim, Germany) from April 2006 to September 2008. Patient preparation, scan protocol and image reconstruction algorithm for 64-slice and dual-source CT scanners are presented in the Appendix Table. The CTCA images were interpreted blinded to the results of the stress test or ICA. Segments distal to a chronic total occlusion were excluded. Segments were scored as having obstructive CAD if there was $\geq 50 \%$ diameter reduction of the lumen by visual assessment.
Invasive coronary angiography (ICA)

Four experienced cardiologists, unaware of the results of the stress test or CTCA, analyzed all coronary segments using a modified 17-segment AHA classification [10]. Segments were visually classified as normal (smooth parallel or tapering borders; visually less than $20 \%$ narrowing) or as having non-significant or significant coronary obstruction (visually more than $20 \%$ narrowing). The stenoses visually scored as having more than $20 \%$ narrowing were quantified by a validated quantitative coronary angiography (QCA) algorithm (CAAS, Pie Medical, Maastricht, the Netherlands) and classified as significant if the lumen diameter reduction exceeded $\geq 50 \%$.

Statistical analysis

Statistical analyses were performed using SPSS (version 12.1. SPSS Inc Chicago Ill. USA). Categorical patients' demographics and characteristics were expressed as numbers and percentages. Continuous variables were expressed as mean (standard deviation).

The pretest probability of having obstructive CAD was calculated using the Duke Clinical Score, which includes types of chest discomfort, age, gender and traditional risk factors [11] and was expressed as mean (standard deviation).

Diagnostic performance and predictive value of exercise bicycle testing, SPECT and CTCA for the diagnosis of obstructive CAD compared to the reference standard was evaluated on a per patient level and expressed as sensitivity, specificity, positive and negative predictive value (PPV and NPV) and their corresponding 95\% confidence intervals, and positive and negative likelihood ratios (LR).

Four comparisons were performed; exercise bicycle testing (conclusive outcome) versus CTCA; exercise bicycle testing (conclusive outcome) and SPECT versus CTCA; exercise bicycle testing (conclusive and inconclusive outcome) versus CTCA; and SPECT versus CTCA. An intention to diagnose design was used: in patients with an inconclusive exercise bicycle test outcome without further testing, the test was scored as a positive outcome. In patients with inconclusive exercise bicycle test outcome with subsequent SPECT, both test results were independently evaluated in the analysis of the diagnostic 
performance of exercise bicycle test (conclusive outcome) and SPECT, respectively.

The area under the receiver operating characteristic (ROC) curve, the c-index, was calculated for EBT (conclusive outcome), EBT (conclusive and inconclusive outcome), EBT (conclusive outcome) and SPECT, SPECT and CTCA.

The McNemar test was performed to compare the sensitivities and specificities for CTCA versus exercise bicycle testing (conclusive outcome), exercise bicycle testing (conclusive outcome) and SPECT, exercise bicycle testing (conclusive and inconclusive outcome) and SPECT, respectively. Additional agreement analyses between the four comparisons were performed with ICA as reference standard.

\section{Results}

Patient demographics $(n=376)$ are listed in Table 1. The mean pretest probability was $61 \pm 30 \%$. A total of $334(89 \%, 334 / 376)$ enrolled patients underwent

Table 1 Patient demographics $(n=376)$

\begin{tabular}{ll}
\hline$N$ & 376 \\
Typical angina & $191(50)$ \\
Atypical angina & $92(25)$ \\
Nonanginal chest pain & $92(25)$ \\
Men & $254(68)$ \\
Age $(\text { years })^{\mathrm{a}}$ & $60.4 \pm 10.0$ \\
BMI $\left(\mathrm{kg} / \mathrm{m}^{2}\right)^{\mathrm{a}}$ & $27.3 \pm 4.1$ \\
Risk factors & \\
Hypertension $^{\mathrm{b}}$ & $170(45)$ \\
Hypercholesterolemia $^{\mathrm{c}}$ & $199(53)$ \\
Diabetes mellitus $^{\mathrm{d}}$ & $56(15)$ \\
Smoker $^{\text {Family history of CAD }}$ & $88(23)$ \\
Obesity $^{\mathrm{f}}$ & $64(17)$ \\
\hline
\end{tabular}

Values are $n(\%)$ unless otherwise indicated

a Mean and standard deviation

b Blood pressure 140/90 $\mathrm{mm} \mathrm{Hg}$ or treatment for hypertension

c Total cholesterol $>180 \mathrm{mg} / \mathrm{dL}$ or treatment for hypercholesterolemia

d Treatment with oral antidiabetic medication or insulin

e Family history of coronary artery disease (CAD), having first- or second-degree relatives with premature CAD (age < 55 years)

${ }^{\mathrm{f}}$ Body mass index (BMI) $30 \mathrm{~kg} / \mathrm{m}^{2}$ exercise bicycle testing and 258 (77\%, 258/334) patients demonstrated a conclusive outcome. In 76 $(23 \%, 76 / 334)$ patients, exercise bicycle testing was inconclusive and 19 (25\%, 19/76) patients were subsequently referred to SPECT. In the remaining 57 $(75 \%, 57 / 76)$ patients with inconclusive exercise bicycle testing without further testing, the test result was scored as positive for CAD. A total of $42(11 \%$, 42/376) enrolled patients were directly referred to SPECT. SPECT outcome was conclusive in all patients.

\section{Diagnostic performance}

The overall prevalence of disease was $73 \%$ (276/ 376). ICA showed absence of disease in $10 \%$ (39/ $376)$, nonsignificant disease in $16 \%$ (61/376), singlevessel disease in $36 \%(135 / 376)$ and multi-vessel disease in $38 \%(141 / 376)$ of patients.

The diagnostic performance for the detection of obstructive CAD on a per patient level with QCA as standard of reference is presented in Table 2.

In patients with a conclusive exercise bicycle testing $(n=258)$, sensitivity of CTCA $(99 \%)$ was significantly $(P$ value $<0.001)$ higher than of exercise bicycle testing (72\%). No significant difference $(P$ value $=0.082)$ was found for specificity between the two modalities: $57 \%$ for exercise bicycle testing and $71 \%$ for CTCA. Including patients with inconclusive exercise bicycle testing $(n=334)$ both sensitivity and specificity were significant $(P$ value $<0.001$ ) higher for CTCA compared to exercise bicycle testing: 100 and 74\% for CTCA, respectively, and 76 and $47 \%$ for exercise bicycle testing (conclusive and inconclusive outcome), respectively. In patients with a conclusive stress test (exercise bicycle testing or SPECT; $n=319)$ the sensitivity was significant higher $(P$ value $<0.001)$ for CTCA (99\%) compared to the stress test, exercise bicycle testing or SPECT (75\%). There was no significant difference $(P$ value $=0.07)$ in specificity between CTCA and stress test: 74 and $61 \%$ respectively. In patients who underwent SPECT $(n=61)$ a significant difference was found for sensitivity $(P \quad$ value $=0.021)$, but not for specificity $(P$ value $=1.0)$ between SPECT and CTCA. Sensitivity was 89 and $98 \%$ for SPECT and CTCA, respectively. Specificity was 77 and $82 \%$ for SPECT and CTCA, respectively. 


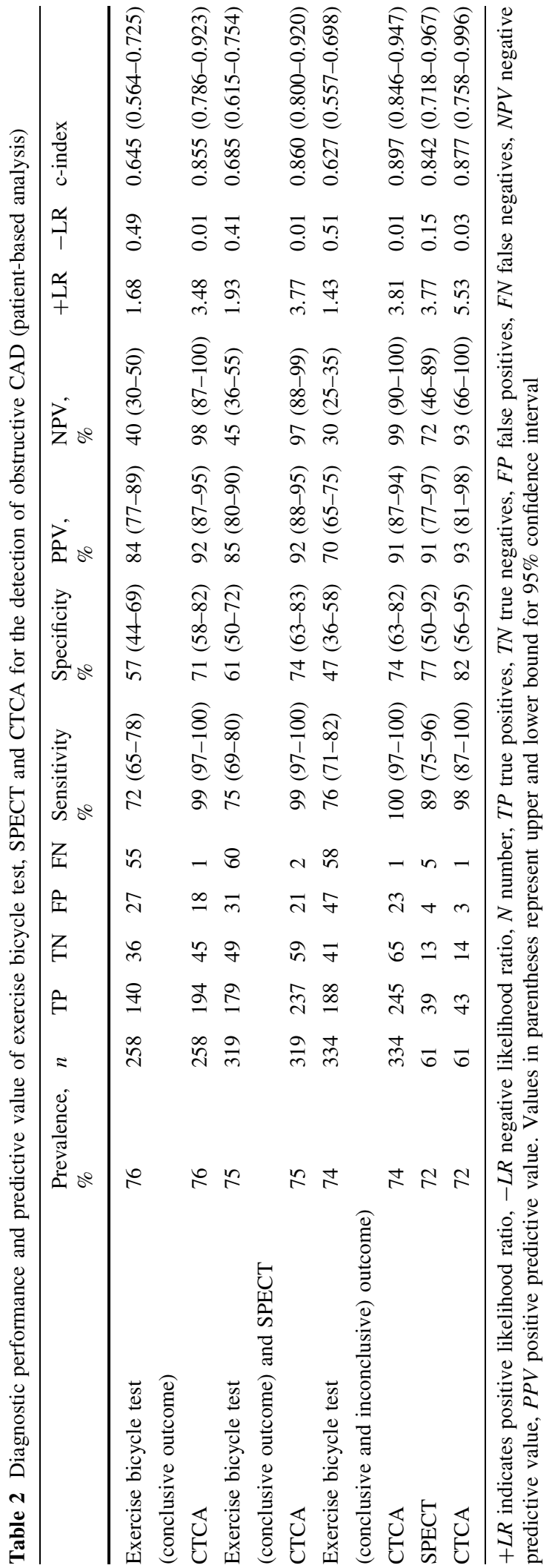

Agreement analysis

The agreement between exercise bicycle testing and CTCA, and SPECT and CTCA, respectively, with ICA as reference standard is presented in Table 3.

\section{Exercise bicycle test and CTCA}

In patients who underwent exercise bicycle testing (conclusive and inconclusive outcome), there was agreement with CTCA in $72 \%$ of patients (239/334). The agreement between a true positive exercise bicycle test and CTCA outcome was 76\% (187/246) in the presence of obstructive CAD at ICA. The agreement between a true negative exercise bicycle test and CTCA outcome was 39\% (35/88) in the absence of obstructive CAD at ICA. In patients with a false negative exercise bicycle test outcome, the presence of obstructive CAD was correctly demonstrated by a positive CTCA in all patients $(100 \%, 58 / 58)$. A negative CT scan correctly ruled out the presence of CAD in $64 \%(30 / 47)$ of patients with a false-positive exercise bicycle test outcome. Exercise bicycle testing correctly identified one patient with obstructive CAD and 6 patients without obstructive CAD, respectively, who were misdiagnosed with CTCA.

\section{SPECT and CTCA}

In patients who underwent SPECT, there was agreement with CTCA in $85 \%$ of patients $(52 / 61)$. The agreement between a true positive SPECT and CTCA was $86 \%$ (38/43) in the presence of obstructive CAD at ICA. The agreement between a true negative SPECT and CTCA was $86 \%(12 / 14)$ in the absence of obstructive CAD at ICA. In 5 patients with a false negative SPECT outcome, the presence of obstructive CAD was correctly demonstrated by a positive CTCA in all patients. A negative CT scan correctly ruled out the presence of CAD in two patients with a falsepositive SPECT outcome. SPECT correctly identified one patient with obstructive CAD who was misdiagnosed with CTCA.

\section{Discussion}

The diagnosis of ischemia remains challenging and extensive effort is invested to improve the 
Table 3 Agreement analysis between CTCA and exercise bicycle test, and CTCA and SPECT (patient based analysis)

\begin{tabular}{|c|c|c|c|c|c|c|}
\hline & $N=258$ & & Exercise & (cons & & \\
\hline & & & $\mathrm{TP}$ & $\mathrm{TN}$ & FP & $\mathrm{FN}$ \\
\hline \multirow[t]{5}{*}{ CTCA } & & $\mathrm{TP}(n=194)$ & 139 & & & 55 \\
\hline & & $\mathrm{TN}(n=45)$ & & 30 & 15 & \\
\hline & & $\mathrm{FP}(n=18)$ & & 6 & 12 & \\
\hline & & $\mathrm{FN}(n=1)$ & 1 & & & 0 \\
\hline & $N=319$ & & Exercise & (con & and SPECT & \\
\hline \multirow[t]{7}{*}{ CTCA } & & & TP & $\mathrm{TN}$ & FP & FN \\
\hline & & $\mathrm{TP}(n=237)$ & 177 & & & 60 \\
\hline & & $\mathrm{TN}(n=59)$ & & 42 & 17 & \\
\hline & & $\mathrm{FP}(n=21)$ & & 7 & 14 & \\
\hline & & $\mathrm{FN}(n=2)$ & 2 & & & 0 \\
\hline & $N=61$ & & SPECT & & & \\
\hline & & & $\mathrm{TP}$ & $\mathrm{TN}$ & FP & FN \\
\hline \multirow[t]{6}{*}{ CTCA } & & $\mathrm{TP}(n=43)$ & 38 & & & 5 \\
\hline & & $\mathrm{TN}(n=14)$ & & 12 & 2 & \\
\hline & & $\mathrm{FP}(n=3)$ & & 1 & 2 & \\
\hline & & $\mathrm{FN}(n=1)$ & 1 & & & 0 \\
\hline & $N=334$ & & Exercise & (cons & nclusive outcome) & \\
\hline & & & $\mathrm{TP}$ & $\mathrm{TN}$ & $\mathrm{FP}$ & FN \\
\hline \multirow[t]{4}{*}{ CTCA } & & $\mathrm{TP}(n=245)$ & 187 & & & 58 \\
\hline & & $\mathrm{TN}(n=65)$ & & 35 & 30 & \\
\hline & & $\mathrm{FP}(n=23)$ & & 6 & 17 & \\
\hline & & $\mathrm{FN}(n=1)$ & 1 & & & 0 \\
\hline
\end{tabular}

$N$ indicates number, $T P$ true positives, $T N$ true negatives, $F P$ false positives, $F N$ false negatives, $N P V$ negative predictive value, SPECT single photon emission computed tomography

noninvasive diagnostic work-up and selection of patients in need for catheterization and possible percutanous catheter treatment. Stress testing prior to percutanous coronary intervention has been associated with shorter hospital stays, and lower rates of revascularization without adverse effects on cardiac death or myocardial infarction [12, 13].

For reasons of availability and costs, traditionally, exercise ECG using treadmill or bicycle testing represents the first-line test to diagnose inducible ischemia in patients presenting with anginal complaints. Exercise bicycle testing should be conducted in patients not taking anti-ischemic drugs, but this may not always be possible or considered safe [7]. Exercise bicycle testing is not of diagnostic value in the presence of a left bundle branch block, paced rhythm and Wolff-Parkinson-White syndrome that prevent reliable evaluation of the ECG changes during stress. Lower specificity and positive predictive value are reported in patients with resting ECG abnormalities, in the presence of left ventricle hypertrophy, electrolyte imbalance, or intraventricular conduction abnormalities and digitalis use. The exercise bicycle test is less sensitive and specific in women, in detecting single vessel disease, right coronary or circumflex artery disease, and in the presence of serial stenoses or extensive collaterals [14].

Nuclear MPI using SPECT has several advantages over exercise bicycle testing including superior diagnostic performance [7], quantification and localization of areas of ischemia, and incremental value for risk stratification [9]. Despite the introduction of $\mathrm{X}$-ray-based attenuation correction and gated scanning, SPECT remains susceptible to a variety of artefacts (respiratory motion, spill-over from gut or liver activity) resulting in low PPV for identifying areas of ischemia in need for revascularization. 
CTCA allows direct visualization of the coronaries and numerous studies have demonstrated a high diagnostic performance of CTCA in selected patient populations [4]. The high NPV (range 95-100\%) permits CTCA to act as reliable gate keeper to discharge patients from further testing. The current drawback of CTCA is the insufficient spatial resolution resulting in lower PPV in particular in the presence of calcium.

We performed a comparison of exercise bicycle testing with CTCA, and SPECT with CTCA, respectively, using ICA as reference standard. The number of inconclusive exercise bicycle test results (23\%) is in line with reported values in literature [15]. The sensitivity of exercise bicycle testing $(72-76 \%)$ is similar to outcome of a large meta-analysis (sensitivity $68 \%$, specificity $77 \%$ ) [16], specificity is lower (47-57\%). Dewey et al. [17] presented comparable results in a head-to-head comparison of exercise ECG and MSCT in 80 patients.

We found that SPECT and CTCA yielded a high diagnostic performance (sensitivity of 89 and $98 \%$; specificity of 77 and $82 \%$ ) that was superior to exercise bicycle testing (sensitivity $76 \%$, specificity $47 \%)$. We observed a reasonable agreement $(76 \%)$ between a true positive exercise bicycle testing and CTCA and only a fair agreement (39\%) was found between a true negative exercise bicycle testing and CTCA. The agreement was good $(86 \%)$ between a true positive SPECT and CTCA and between a true negative SPECT and CTCA.

Our results are partly in line with reported studies on comparison between SPECT and CTCA that showed a good agreement (range 86-96\%) in the absence of obstructive CAD [18-20]. However, these studies demonstrated a lower agreement (range 50-67\%) for the detection of lesions with CTCA that induced myocardial perfusion defects. The discrepancies in agreement should be interpreted in the context of the pretest risk of disease and the standard of reference. Furthermore, the standard of reference ICA was not used in all patients with the exception of one study [18].

Which modality will represent the first-line test in the diagnostic work-up of patients with stable angina is largely unknown and depends on its relative costs and availability, the pretest risk of disease in the cohort to be examined, and the number of patients that can be identified as not needing further evaluation. Notably, with the advent of noninvasive cardiac imaging testing, cumulative patient dose may become considerably high.

The exercise bicycle test is widely availably, not costly and safe compared to SPECT and CTCA, and despite its inferior diagnostic performance, may represent the first line test in patients with a low pretest risk. SPECT and CTCA have superior diagnostic performance, but are associated with higher costs and radiation exposure, and may represent more appropriate initial tests in patients with an intermediate to high pretest risk.

In patients with stable angina, both normal CTCA and SPECT have a low risk of hard coronary events (cardiac death or nonfatal myocardial infarction) obviating the need for catheterization or further testing [5, 9].

SPECT as initial test may identify jeopardized ischemic myocardium on a per patient level [38], which fulfills the recommendations of the guidelines prior to revascularization. However, several studies indicated that SPECT only moderately guides revascularization treatment of the ischemia-related vessel [21-23]. CTCA could add valuable information to MPI by allocating perfusion defects to specific epicardial coronary vessels. However, there is a known discrepancy in hemodynamic significance of intermediate lesions with a luminal diameter stenosis between 40 and $70 \%$ [18, 24]. Previous reports have demonstrated that the anatomical assessment of a stenosis as determined by ICA or CTCA correlates poorly with the hemodynamic significance of intermediate coronary stenoses as measured by invasive fractional flow reserve (FFR) [24-26]. Luminal diameter stenosis measurement does not always reflect coronary artery resistance as it neglects specific lesion characteristics, vasomotor tone or presence of coronary collateral flow that may significantly affect myocardial perfusion [27]. Individual variations in coronary anatomy may also contribute to inadequate allocation of perfusion defects to corresponding coronary arteries [20].

On the other hand, in case of multivessel disease lack of perfusion defects at SPECT in patients with obstructive lesions at ICA may be attributed to a balanced reduction in myocardial perfusion owing to the compromised coronary vasodilator reserve in territories supplied by angiography stenoses and thereby reducing the heterogeneity of flow between 
"normal" and "abnormal" zones [28]. In addition, the absence of epicardial coronary stenosis in the presence of a perfusion defect may be caused by microvascular dysfunction. At present, CTCA serves as a reliable rule-out test for the presence of significant CAD. Combined noninvasive anatomical and functional imaging may best identify patients who are likely to benefit most from secondary prevention and optimal medical therapy (no or mild ischemia present) or who may be candidates for coronary revascularization (moderate to severe ischemia present).

The potential of 2D/3D image fusion techniques or hybrid scanners (SPECT/CT) to correctly link coronary stenoses at CTCA to perfusion defects to resolve inadequate allocation of perfusion defects is now being explored. The clinical application of cardiac hybrid imaging was hampered due to the associated high radiation exposure to the patient (up to $40 \mathrm{mSv}$ ) [48] but technical advances now permit hybrid imaging below $3 \mathrm{mSv}$ with low-dose CTCA using prospective ECG triggering combined with stressonly SPECT [29].

Further investigation is needed to evaluate the diagnostic performance of SPECT/CT to accurately allocate and guide treatment of the target vessel.

\section{Limitations}

For the comparison SPECT versus CTCA, analyses were limited to small sample size.
The possibility of referral bias may occur when patients are referred to the reference test based on the results of the noninvasive test under investigation. Sensitivity may be inflated and specificity deflated if patients with a positive test result are more likely to be verified.

We performed a single center study and the influence of local practice and referral patterns, technical capabilities for performance of each modality may vary from institution to institution.

\section{Conclusions}

SPECT and CTCA yielded a higher diagnostic performance compared to traditional exercise bicycle testinging for the detection and rule out of obstructive CAD in patients with stable angina. Future studies are needed to evaluate the clinical value of traditional noninvasive diagnostic tests compared to new test such as CTCA or hybrid CTCA/SPECT taking into account the pretest risk, availability, radiation exposure and cost-effectiveness.

Open Access This article is distributed under the terms of the Creative Commons Attribution Noncommercial License which permits any noncommercial use, distribution, and reproduction in any medium, provided the original author(s) and source are credited.

\section{Appendix}

See Table 4.
Table 4 CTCA protocol

\begin{tabular}{lll}
\hline Patient preparation & $64-$ slice $\mathrm{CT}^{\mathrm{a}}$ & Dual-source $\mathrm{CT}^{\mathrm{b}}$ \\
Betablockers (dose, oral) & Yes & No \\
Lorazepam (dose, oral) & Yes & No \\
Nitroglycirine (0.4 mg/dose, sublingual) & No & Yes \\
Scan parameters & & \\
No. of tubes & 1 & 2 \\
No. of detectors & $32 \times 2-64$ & $32 \times 2-64$ \\
Collimation (mm) & $32 \times 2 \times 0.6 \mathrm{~mm}^{\mathrm{c}}$ & $32 \times 2 \times 0.6 \mathrm{~mm}^{\mathrm{c}}$ \\
Gantry rotation time (ms) & 330 & 330 \\
Effective temporal resolution (ms) & $165 \mathrm{~ms}^{\mathrm{d}}$ & $83 \mathrm{~ms}^{\mathrm{d}}$ \\
Pitch & 0.20 & $0.20-0.53$ \\
$\mathrm{kV}$ & 120 & 120 \\
Full tube current (mA) & $800-900$ & 625 \\
ECG pulsing & Yes & Yes \\
\hline
\end{tabular}


Table 4 continued

a Sensation ${ }^{\circledR}$, Siemens,
Forchheim, Germany
b Definition ${ }^{\circledR}$, Siemens,
Forchheim, Germany
c Z-sharp ${ }^{\circledR}$ Technology
Siemens, Forchheim,
Germany
d Single-segmental
reconstruction algorithm

\begin{tabular}{lll}
\hline Scan time range $(\mathrm{s})$ & $15-20$ & $5-15$ \\
Bolus tracking & Yes & Yes \\
Contrast volume $(\mathrm{mL})$ & $50-120$ & $60-100$ \\
Contrast agent $(\mathrm{mgl} / \mathrm{mL})$ & 400 & 370 \\
Saline chaser volume $(\mathrm{mL})$ & 40 & 40 \\
Injection rate $(\mathrm{mL} / \mathrm{sec})$ & $4.0-5.0$ & 5.5 \\
Image reconstruction & & \\
Effective slice width $(\mathrm{mm})$ & 0.75 & 0.75 \\
Reconstruction interval $(\mathrm{mm})$ & 0.4 & 0.4 \\
Field of view $(\mathrm{mm})$ & 180 & 180 \\
Convolution filter & Medium/sharp & Medium/sharp \\
\hline
\end{tabular}

\section{References}

1. Daly CA, Clemens F, Sendon JL et al (2005) The clinical characteristics and investigations planned in patients with stable angina presenting to cardiologists in Europe: from the Euro Heart Survey of Stable Angina. Eur Heart J 26:996-1010

2. Sekhri N, Feder GS, Junghans C et al (2008) Incremental prognostic value of the exercise electrocardiogram in the initial assessment of patients with suspected angina: cohort study. Bmj 337:a2240

3. Underwood SR, Anagnostopoulos C, Cerqueira M et al (2004) Myocardial perfusion scintigraphy: the evidence. Eur J Nucl Med Mol Imaging 31:261-291

4. Abdulla J, Abildstrom SZ, Gotzsche O, Christensen E, Kober L, Torp-Pedersen C (2007) 64-multislice detector computed tomography coronary angiography as potential alternative to conventional coronary angiography: a systematic review and meta-analysis. Eur Heart J 28:3042-3050

5. Min JK, Shaw LJ, Devereux RB et al (2007) Prognostic value of multidetector coronary computed tomographic angiography for prediction of all-cause mortality. J Am Coll Cardiol 50:1161-1170

6. Ostrom MP, Gopal A, Ahmadi N et al (2008) Mortality incidence and the severity of coronary atherosclerosis assessed by computed tomography angiography. J Am Coll Cardiol 52:1335-1343

7. Fox K, Garcia MA, Ardissino D et al (2006) Guidelines on the management of stable angina pectoris: executive summary: the task force on the management of stable angina pectoris of the European society of cardiology. Eur Heart J 27:1341-1381

8. Rubinshtein R, Halon DA, Gaspar T et al (2007) Usefulness of 64-slice multidetector computed tomography in diagnostic triage of patients with chest pain and negative or nondiagnostic exercise treadmill test result. Am J Cardiol 99:925-929

9. Schinkel AF, Elhendy A, Van Domburg RT et al (2002) Long-term prognostic value of dobutamine stress $99 \mathrm{mTc}-$ sestamibi SPECT: single-center experience with 8-year follow-up. Radiology 225:701-706

10. Austen WG, Edwards JE, Frye RL et al (1975) A reporting system on patients evaluated for coronary artery disease.
Report of the Ad Hoc committee for grading of coronary artery disease, council on cardiovascular surgery, american heart association. Circulation 51:5-40

11. Pryor DB, Shaw L, McCants CB et al (1993) Value of the history and physical in identifying patients at increased risk for coronary artery disease. Ann Intern Med 118:81-90

12. Shaw LJ, Hachamovitch R, Berman DS et al (1999) The economic consequences of available diagnostic and prognostic strategies for the evaluation of stable angina patients: an observational assessment of the value of precatheterization ischemia. Economics of Noninvasive Diagnosis (END) Multicenter Study Group. J Am Coll Cardiol 33:661-669

13. Topol EJ, Ellis SG, Cosgrove DM et al (1993) Analysis of coronary angioplasty practice in the United States with an insurance-claims data base. Circulation 87:1489-1497

14. Hlatky MA, Pryor DB, Harrell FE Jr, Califf RM, Mark DB, Rosati RA (1984) Factors affecting sensitivity and specificity of exercise electrocardiography. Multivariable analysis. Am J Med 77:64-71

15. Philbrick JT, Horwitz RI, Feinstein AR, Langou RA, Chandler JP (1982) The limited spectrum of patients studied in exercise test research. Analyzing the tip of the iceberg. Jama 248:2467-2470

16. Gibbons RJ, Balady GJ, Bricker JT et al (2002) ACC/AHA 2002 guideline update for exercise testing: summary article: a report of the American College of Cardiology/ American Heart Association Task Force on Practice Guidelines (Committee to Update the 1997 Exercise Testing Guidelines). Circulation 106:1883-1892

17. Dewey M, Dubel HP, Schink T, Baumann G, Hamm B (2007) Head-to-head comparison of multislice computed tomography and exercise electrocardiography for diagnosis of coronary artery disease. Eur Heart J 28:2485-2490

18. Gaemperli O, Schepis T, Valenta I et al (2008) Functionally relevant coronary artery disease: comparison of 64-section CT angiography with myocardial perfusion SPECT. Radiology 248:414-423

19. Hacker M, Jakobs T, Hack N et al (2007) Sixty-four slice spiral CT angiography does not predict the functional relevance of coronary artery stenoses in patients with stable angina. Eur J Nucl Med Mol Imaging 34:4-10

20. Schuijf JD, Wijns W, Jukema JW et al (2006) Relationship between noninvasive coronary angiography with multi- 
slice computed tomography and myocardial perfusion imaging. J Am Coll Cardiol 48:2508-2514

21. Christian TF, Miller TD, Bailey KR, Gibbons RJ (1992) Noninvasive identification of severe coronary artery disease using exercise tomographic thallium-201 imaging. Am J Cardiol 70:14-20

22. Hacker M, Rieber J, Schmid R et al (2005) Comparison of Tc-99m sestamibi SPECT with fractional flow reserve in patients with intermediate coronary artery stenoses. J Nucl Cardiol 12:645-654

23. Travin MI, Katz MS, Moulton AW, Miele NJ, Sharaf BL, Johnson LL (2000) Accuracy of dipyridamole SPECT imaging in identifying individual coronary stenoses and multivessel disease in women versus men. J Nucl Cardiol 7:213-220

24. Meijboom WB, Van Mieghem CA, van Pelt N et al (2008) Comprehensive assessment of coronary artery stenoses: computed tomography coronary angiography versus conventional coronary angiography and correlation with fractional flow reserve in patients with stable angina. J Am Coll Cardiol 52:636-643
25. Heller LI, Cates C, Popma J et al (1997) Intracoronary Doppler assessment of moderate coronary artery disease: comparison with $201 \mathrm{Tl}$ imaging and coronary angiography. FACTS Study Group. Circulation 96:484-490

26. Berger A, Botman KJ, MacCarthy PA et al (2005) Longterm clinical outcome after fractional flow reserve-guided percutaneous coronary intervention in patients with multivessel disease. J Am Coll Cardiol 46:438-442

27. Gould KL (1988) Identifying and measuring severity of coronary artery stenosis. Quantitative coronary arteriography and positron emission tomography. Circulation 78:237-245

28. Uren NG, Melin JA, De Bruyne B, Wijns W, Baudhuin T, Camici PG (1994) Relation between myocardial blood flow and the severity of coronary-artery stenosis. N Engl J Med 330:1782-1788

29. Herzog BA, Husmann L, Landmesser U, Kaufmann PA (2009) Low-dose computed tomography coronary angiography and myocardial perfusion imaging: cardiac hybrid imaging below 3 mSv. Eur Heart J 30:644 